\title{
Alterações de cílios no Hospital das Clínicas da Faculdade de Medicina de Ribeirão Preto-USP
}

\author{
Eyelash abnormalities at the Hospital of the School of Medicine of Ribeirão Preto
}

\author{
Fabiana Athayde Martins Araújo ${ }^{1}$ \\ Antonio Augusto Velasco de Cruz ${ }^{2}$
}

${ }^{1}$ Médica, pós-graduanda - Departamento de Oftalmologia, Otorrinolaringologia e Cabeça e Pescoço da Faculdade de Medicina de Ribeirão Preto - USP.

${ }^{2}$ Prof. Associado do Departamento de Oftalmologia, Otorrinolaringologia e Cabeça e Pescoço da Faculdade de Medicina de Ribeirão Preto - USP.

Essa pesquisa contou com apoio financeiro da CAPES

Endereço para correspondência: Departamento de Oftalmologia, Otorrinolaringologia e Cirurgia de Cabeça e Pescoço, Hospital das Clínicas - Av. Bandeirantes, 3900 - Ribeirão Preto (SP) CEP 14048-900.

E-mail: aavecruz@fmrp.usp.br - Fax 16-6022860

Recebido para publicação em 10.05.2001

Aceito para publicação em 12.12.2001

Nota Editorial: Pela análise deste trabalho e por sua anuência sobre a divulgação desta nota, agradecemos ao Dr. Waldir Martins Portellinha.

\section{RESUMO}

Objetivo: Sistematizar (classificar e caracterizar) os problemas ciliares vistos no setor de oculoplástica da Faculdade de Medicina de Ribeirão Preto - USP. Métodos: Avaliaram-se consecutiva e prospectivamente 59 pacientes do setor de oculoplástica da Faculdade de Medicina de Ribeirão Preto - USP, no ano de 1999, com doenças ciliares, excluindo-se aqueles com tratamento prévio que não fosse epilação. Foi investigado presença de doença associada (local e/ou sistêmica), queixa e duração dos sinais e sintomas, presença de alterações palpebrais e de alterações da superfície ocular. Os cílios patológicos foram caracterizados em relação à cor, espessura e localização e lateralidade da pálpebra. Resultados: A principal doença ciliar encontrada foi a triquíase, aparecendo em 56 pacientes ou $94,9 \%$ dos casos. Um caso raro encontrado foi o de um cílio ectópico incarnatum internum. O tracoma foi a principal etiologia associada à triquíase. A triquíase tracomatosa diferenciou-se da não-tracomatosa quanto ao número de segmentos palpebrais atingidos, lateralidade, pálpebra afetada (superior e inferior) e magnitude do dano à superfície ocular. Alterações de tamanho, cor e espessura dos cílios patológicos foram verificadas tanto na triquíase tracomatosa como não-tracomatosa. Conclusões: A triquíase tracomatosa ainda é o principal problema ciliar no HCFMRP-USP. Alterações de cor e espessura ciliar são vistas tanto na triquíase tracomatosa como na não-tracomatosa e, portanto, não são critérios válidos para diferenciar distiquíase adquirida de triquíase.

Descritores: Cílios; Tracoma; Doenças palpebrais; Pálpebras

\section{INTRODUÇÃO}

É curioso notar que, embora o espectro de alterações ciliares seja considerável e extremamente freqüente na prática oftalmológica, a literatura sobre o assunto é esparsa e confusa. A terminologia usada para a denominação de problemas ciliares não é consensual entre os especialistas oculoplásticos e alguns termos, como ptose ciliar, usados na literatura americana ${ }^{(1-2)}$, não são aceitos de maneira unânime.

O objetivo que norteou a realização desse trabalho foi o desejo de sistematizar os problemas ciliares vistos no setor de oculoplástica do Departamento de Oftalmologia e Otorrinolaringologia da Faculdade de Medicina de Ribeirão Preto - USP. A importância de um levantamento desse tipo está ligada não só ao aspecto didático, de ensino para residentes, como também ao plano assistencial, na medida que a correta caracterização dos diferentes tipos de problemas ciliares permite uma melhor adequação das propostas terapêuticas. Para esse fim, foi usada a classificação de anoma- 
lias ciliares adotada no referido setor e que, face a falta de uniformidade terminológica, encontra-se abaixo detalhada.

\section{Classificação de anomalias ciliares}

\section{1 - Anomalias constitucionais}

\section{1 - De número}

1.1.1 - Politricose: aumento do número de cílios ${ }^{(3)}$.

1.1.2 - Madarose: perda ou queda de cílios, levando à diminuição do número de cílios na pálpebra. Tem o mesmo significado de alopécia ciliar quando a madarose é total ${ }^{(3)}$. Quando parcial, o termo madarose é mais utilizado. A madarose é usualmente a expressão de um problema orgânico, entretanto, há casos funcionais nos quais a perda de cílios decorre de auto-agressão. É a tricotilomania ${ }^{(4)}$.

\section{2 - De tamanho}

1.2.1 - Tricomegalia: crescimento excessivo, acarretando aumento no comprimento final do cílio( ${ }^{(3)}$. Essa condição talvez decorra de uma desregulação do ciclo de crescimento ciliar ${ }^{(5)}$. A anomalia oposta, cílios pequenos, não é usualmente distinguida com um termo específico. A tricomegalia pode fazer parte ou não da hipertricose, que é uma condição congênita ou adquirida caracterizada por aumento do tamanho dos pêlos de todo o corpo $^{(3)}$.

\section{3 - De orientação ou direcionamento}

1.3.1 - Triquíase: esse termo denota alteração de orientação na qual os cílios perdem o direcionamento normal (afastando-se do olho) e tendem a tocar a superfície ocular. É de natureza cicatricial, sempre adquirida, podendo ser mais ou menos limita$\mathrm{da}^{(6,3,7-8)}$. Alguns autores classificam a magnitude dessa patologia segundo o número de cílios envolvidos. Assim, na triquíase maior, mais do que 5 cílios tocam o olho e na triquíase menor 5 cílios ou menos se encontram mal posicionados ${ }^{(9-11)}$.

1.3.2 - Ptose ciliar: expressão recente, introduzida na literatura americana para denotar anomalia de orientação, caracterizada por abaixamento global dos cílios ou de importantes segmentos dos mesmos ${ }^{(1-2)}$. A ptose ciliar é sempre um problema de atonia da lamela anterior, causando perda da função do músculo de Riolan e, portanto, abaixamento mais ou menos acentuado dos cílios. É um termo aplicado basicamente para a pálpebra superior.

1.3.3 - Retração ciliar: termo usado para descrever a condição oposta à ptose ciliar, ou seja, retração da lamela anterior e conseqüente exagero na curvatura ciliar. Também se refere à pálpebra superior ${ }^{(12)}$.

1.3.4 - Cilia incarnata ou pili incarnati: anomalia de crescimento caracterizada pelo direcionamento horizontal do cílio que permanece sob a epiderme. Quando o cílio aparece sob a conjuntiva tarsal, fala-se em cilium incarnatum internum. Mais raramente, o crescimento pode-se dar para dentro da pele, condição denominada de cilium inversum ${ }^{(3)}$.

1.3.5 - Pili torti: condição congênita caracterizada pela presença de cílios tortuosos na pálpebra superior e inferior ${ }^{(3)}$.

1.4 - De pigmentação

1.4.1 - Poliose: perda da melanina ciliar levando à descolo- ração ou mesmo embranquecimento do cílio. Pode ser congênita ou adquirida, superior ou inferior ${ }^{(3)}$.

\section{5 - De espessura}

1.5.1 - Afinamento: a presença de cílios mais finos que o usual, do tipo lanugo, não é distinguida por um termo específico. Neste trabalho utilizar-se-á o termo "mais fino", ou, simplesmente, cílio fino.

\section{6 - De posição}

1.6.1 - Cílio ectópico: os raros casos descritos foram de tufos ciliares originados da lamela anterior em posição francamente anômala ${ }^{(13)}$.

\section{7 - Do número de fileiras}

1.7.1 - Distiquíase: essa condição designa uma dupla fileira de cílios, anormalmente posicionada atrás da linha cinzenta, usualmente, nos orifícios das glândulas de Meibomius. Poucas palavras em oftalmologia são mais deturpadas que a distiquíase. Ela é freqüentemente confundida com triquíase e, às vezes, pronunciada erroneamente como "distriquíase". Parte dessa confusão certamente advém do fato de o prefixo da palavra não ser o latino dis (dificuldade), mas, sim, o grego $d i$ (duplo). O sufixo grego stichos completa a palavra, significando fileira ${ }^{(3)}$. Para alguns, como Duke-Elder, o termo deveria ser aplicado unicamente para casos congênitos ${ }^{(3)}$. Modernamente, no entanto, usa-se a expressão distiquíase adquirida para designar o crescimento de neocílios na lamela posterior em pacientes com seqüelas de patologia indutora de metaplasia das glândulas de Meibomius, como tipicamente ocorre na síndrome de Steven-Johnson ${ }^{(6)}$. Os termos "tristiquíase e tetrastiquíase" são usados para designar condições congênitas caracterizadas pela presença de, respectivamente, três e quatro fileiras anômalas de cílios ${ }^{(14)}$.

\section{2 - Parasitoses}

Os cílios podem ser parasitados por fungos (Trichorrhexis nodosa e Monilethrix) ${ }^{(3)}$ ou mais comumente por piolhos, dentre os quais o Pediculus humanus capitis (piolho vulgar do couro cabeludo) e o Phthirus pubis, que tipicamente é sexualmente transmitido. Ambas as espécies podem, eventualmente, infestar os cílios em casos de parasitose intensa ${ }^{(15)}$.

\section{3 - Tumores}

Tumores de origem ciliar incluem: tricoepitelioma, tricoadenoma, tricofoliculoma, triquilemoma e pilomatrixoma. Todos são benignos e extremamente $\operatorname{raros}^{(16)}$.

\section{MÉTODOS}

Foram avaliados, prospectivamente, todos os pacientes que procuraram o setor de Plástica Ocular do Serviço de Oftalmologia da Faculdade de Medicina de Ribeirão Preto - USP com patologias ciliares diagnosticadas durante o ano de 1999. O único critério de entrada no estudo foi a não realização de tratamento prévio, exceto eventual epilação. A amostra foi constituída por 59 pacientes. 


\begin{tabular}{|c|c|c|c|c|c|}
\hline \multicolumn{2}{|c|}{ Triquíase } & \multirow{2}{*}{$\begin{array}{l}\text { Cílio ectópico } \\
\text { incarnatum internum }\end{array}$} & \multirow{2}{*}{$\begin{array}{l}\text { Madarose + Poliose } \\
\text { (Lupus discóide) }\end{array}$} & \multirow{2}{*}{$\begin{array}{c}\text { Poliose + } \\
\text { (doença de Graves) }\end{array}$} & \multirow[t]{2}{*}{ Total } \\
\hline Isolada & Associada & & & & \\
\hline $45(76,3 \%)$ & $11(18,6 \%)$ & $1(1,7 \%)$ & $1(1,7 \%)$ & $1(1,7 \%)$ & 59 \\
\hline
\end{tabular}

Para todos os pacientes, foram coletados dados pessoais como idade, sexo e cor. Foram também avaliados parâmetros relacionados ao problema ciliar como presença de doença associada (local e/ou sistêmica), queixa e duração dos sinais e sintomas, presença de alterações palpebrais relevantes como entrópio e ectrópio e de alterações de superfície ocular. Os cílios patológicos foram caracterizados em relação a cor, espessura e localização na pálpebra, sendo também detalhada qual a pálpebra afetada e qual segmento palpebral afetado (medial, central, lateral ou qualquer combinação destes).

Todos os pacientes tiveram o olho direito e esquerdo fotografados com uma máquina fotográfica analógica marca Nikon modelo N6006, equipada com uma lente macro 1:1, $105 \mathrm{~mm}$, Lester A. Dine, em posição primária do olhar, sem queixeira, utilizando flash e filmes Kodak ${ }^{\circledR}$, iso 100, com abertura máxima do diafragma da lente (de 32), a fim de registrar com maiores detalhes os cílios patológicos e possibilitar posterior análise dos dados.

A análise dos dados foi realizada com técnicas de estatística descritiva clássica. Comparações foram efetuadas pelo teste do Qui-quadrado ou exato de Fischer.

\section{RESULTADOS}

Dos 59 pacientes avaliados, 34 eram do sexo feminino e 25 do sexo masculino, com idades variando entre 4 e 92 anos e média de 66,8 anos (Figura 1). Quanto à sintomatologia, 46 pacientes $(77,97 \%)$ foram polissintomáticos, 8 monossintomáticos e 5 assintomáticos. A principal queixa dos monossintomáticos foi a sensação de corpo estranho ( $75 \%$ dos casos), seguida de lacrimejamento $(12,5 \%)$ e de outras queixas como dor, ardor, prurido, olho vermelho. Nos polissintomáticos, as queixas mais freqüentes foram combinações das queixas acima, associadas ou não à queixa de secreção e olho seco.

A duração desses sintomas era variada e 3 pacientes $(5,1 \%)$ não sabiam precisar há quanto tempo eram sintomáticos. Queixas recentes (menos de 1 ano), foram encontradas em apenas $16,95 \%$ dos pacientes. Na maioria dos casos, a duração dos sintomas esteve entre 1 e 10 anos $(37,29 \%)$.

A tabela 1 relaciona as principais alterações ciliares diagnosticadas.

Inicialmente, vale ressaltar o caso de cílio ectópico incarnatum internum pela sua raridade (Figura 2).

Dos 11 casos em que a triquíase apareceu associadamente a outra alteração ciliar, a poliose, isoladamente, foi a anomalia mais freqüente ( 9 casos). Os outros 2 casos foram, 1 caso de distiquíase adquirida e outro de ptose ciliar + poliose.
A tabela 2 detalha a etiologia dos 56 casos de triquíase diagnosticados.

Dos 3 casos em que a triquíase tracomatosa esteve associada a outras patologias, em 2 deles a associação foi com hanseníase e um com psoríase.

É interessante notar que houve algumas diferenças importantes da triquíase tracomatosa e da não-tracomatosa em relação à pálpebra acometida e à extensão do acometimento palpebral (Tabelas 4 e 5). Assim, a triquíase tracomatosa foi significativamente mais extensa e predominantemente superior.

Já em relação a lateralidade não houve diferença significativa entre a triquíase tracomatosa e não-tracomatosa como mostra a tabela 3 .

\begin{tabular}{|lllll|}
\hline \multicolumn{4}{|c|}{ Tabela 2. Etiologia dos $\mathbf{5 6}$ casos de triquíase diagnosticados } \\
\multicolumn{2}{|c|}{ Tracoma } & $\begin{array}{c}\text { Blefarite/ } \\
\text { Meibomite }\end{array}$ & $\begin{array}{l}\text { Indeter- } \\
\text { minada }\end{array}$ & Total \\
\hline Isolado & Associado & Meibor & \\
$23(41,0 \%)$ & $3(5,4 \%)$ & $22(39,3 \%)$ & $8(14,3 \%)$ & $56(100 \%)$ \\
\hline
\end{tabular}

\begin{tabular}{|lccc|}
\hline \multicolumn{4}{|l}{ Tabela 3. Lateralidade da triquíase tracomatosa e não-tracomatosa } \\
\hline Lateralidade & $\begin{array}{c}\text { Triquíase } \\
\text { tracomatosa }\end{array}$ & $\begin{array}{c}\text { Triquíase } \\
\text { não-tracomatosa }\end{array}$ & Total \\
Bilateral & $20^{*}$ & $20^{*}$ & $40(71,4 \%)$ \\
Unilateral & $6^{*}$ & $10^{*}$ & $16(28,6 \%)$ \\
Total & 26 & 30 & $56(100 \%)$ \\
${ }^{*} \Pi^{2}=0,72, p=0,4 ;$ não significativo & & \\
\hline
\end{tabular}

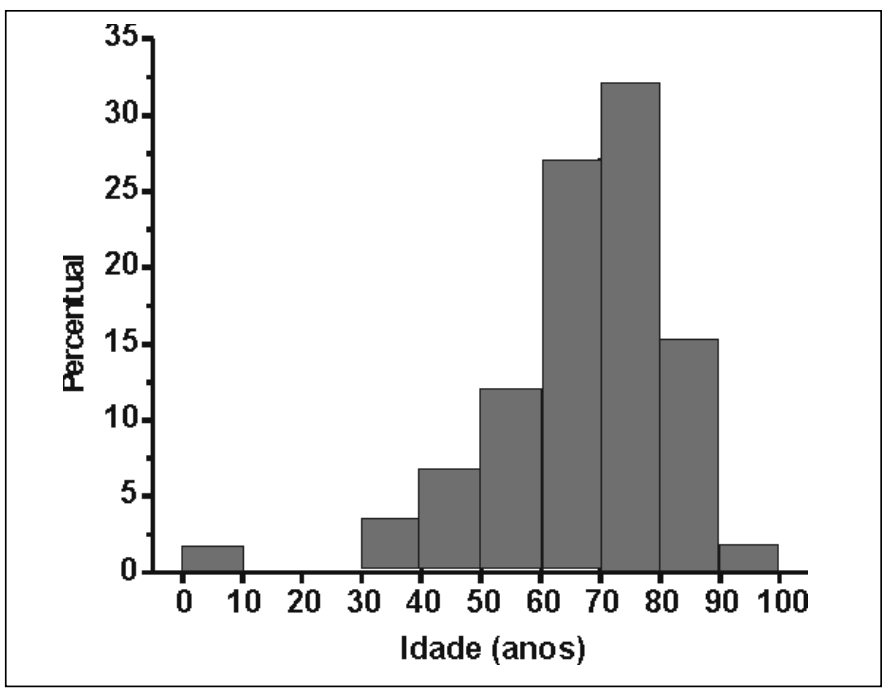

Figura 1 - Distribuição das idades de pacientes com anomalias ciliares diagnosticadas no HCFMRP-USP durante o ano de 1999 


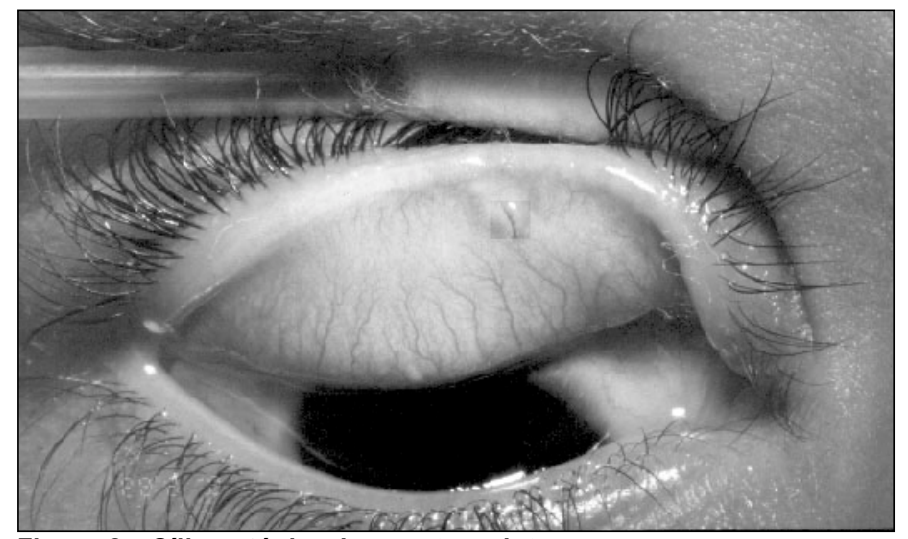

Figura 2 - Cílio ectópico incarnaturm internum

Tabela 4. Acometimento palpebral na triquíase tracomatosa e não-tracomatosa

\begin{tabular}{lccc}
$\begin{array}{l}\text { Pálpebra } \\
\text { acometida }\end{array}$ & $\begin{array}{c}\text { Triquíase } \\
\text { tracomatosa }\end{array}$ & $\begin{array}{c}\text { Triquíase } \\
\text { não-tracomatosa }\end{array}$ & Total \\
Superior & $16^{*}$ & $2^{*}$ & 18 \\
Superior e Inferior & 6 & 4 & 10 \\
Inferior & $4^{*}$ & $24^{*}$ & 28 \\
Total & 26 & 30 & 56 \\
*Acometimento pálpebra superior versus pálpebra inferior, teste de Fischer, \\
p $<0,00001$; significativo & & \\
\hline
\end{tabular}

Tabela 5 . Extensão do acometimento palpebral na triquíase tracomatosa e não-tracomatosa

\begin{tabular}{|lccc|}
$\begin{array}{l}\text { Segmentos palpebrais } \\
\text { acometidos }\end{array}$ & $\begin{array}{c}\text { Triquíase } \\
\text { tracomatosa }\end{array}$ & $\begin{array}{c}\text { Triquíase } \\
\text { não-tracomatosa }\end{array}$ & Total \\
1 & $8^{*}$ & $24^{*}$ & 32 \\
2 ou 3 & $18^{*}$ & $6^{*}$ & 24 \\
Total & 26 & 30 & 56 \\
$\Pi^{2}=13,78, p=0,0002$; significativo & & \\
\hline
\end{tabular}

A triquíase esteve associada a dano corneano em 84 dos 96 olhos acometidos, ou seja, $87,5 \%$ dos casos. A ceratite puntata foi a alteração corneana mais freqüentemente encontrada ( 66 dos 96 olhos com triquíase - 68,75\%). Outras alterações corneanas encontradas foram fossetas límbicas (42 olhos - $43,75 \%$ ), pannus ( 24 olhos $-25 \%$ ) e opacidades (14 olhos - $14,58 \%$ ).

Alterações da superfície ocular foram encontradas na grande maioria $(87,5 \%)$ dos 96 olhos dos pacientes com triquíase. A figura 3 indica que essas alterações também foram tão mais freqüentes nos olhos com tracoma, o que dispensa a realização de teste estatístico.

Em relação às características morfológicas dos cílios em triquíase, dos 26 pacientes com tracoma, 20 (76,9\%) apresentaram pelo menos um cílio patológico mais claro e/ou mais fino (Figura 4). Dos 30 pacientes sem associação com tracoma, essa relação foi de $25(83,3 \%)$ com pelo menos um cílio mais claro e $26(86,7 \%)$ com pelo menos um cílio mais fino (Figura 5).
Finalmente, dos 56 pacientes com triquíase, 25 apresentaram algum tipo de alteração palpebral. Desses, apenas 1 caso não estava associado ao tracoma. Tratava-se de 1 paciente com ectrópio paralítico. Todos os outros 23 casos eram de entrópio cicatricial devido ao tracoma, sendo que deles, 3 pacientes também apresentavam entrópio de pálpebra inferior.

\section{DISCUSSÃO}

Apesar da grande diversidade de patologias ciliares existentes, foi pequena a variedade das anomalias ciliares encontradas em nosso serviço, pois apenas uma condição - a triquíase - foi responsável isoladamente, ou em associação a outras patologias, por $94,9 \%$ dos casos analisados no presente estudo. Os $5,1 \%$ de casos restantes distribuíram-se em somente 3 outras condições (cílio ectópico incarnatum internum, poliose e madarose). Isso pode ter decorrido do fato de algumas anomalias ciliares serem extremamente raras e/ou a maioria delas ser assintomática, não levando o paciente ao oftalmologista. De maneira similar, pode-se aventar a hipótese de a triquíase ser altamente sintomática, o que leva os pacientes portadores dessa condição a procurarem auxílio médico com mais freqüência.

Assim, os dados epidemiológicos levantados no presente estudo referem-se, basicamente à triquíase. A análise da figura 1 (distribuição dos pacientes portadores de anomalias ciliares, segundo a faixa etária) revela que, se o caso de cílio ectópico incarnatum não for levado em consideração por ser uma anomalia congênita, o intervalo de classe modal foi de 70 a 80 anos. Os problemas ciliares adquiridos foram, portanto, virtualmente inexistentes antes dos 30 anos e a prevalência desse tipo de patologia aumentou significativamente com a idade. Esses dados são corroborados por outros trabalhos nos quais a faixa etária encontrada foi semelhante ${ }^{(9-11,17-18)}$.

Em relação à sintomatologia, a maioria dos pacientes com patologia ciliar foi sintomática (91,5\%), e o principal sintoma, quer nos pacientes mono ou polissintomáticos, foi a sensação de corpo estranho. Esse sintoma também foi a queixa mais freqüente de pacientes com triquíase em outros estudos ${ }^{(10-11)}$. Outras queixas menos referidas foram lacrimejamento, secreção, sensação de olho seco e ardor.

Foi freqüente a duração prolongada das queixas (maior que 20 anos, em 33,9\%), indicando a permanência dos problemas ciliares por longos períodos de tempo. Nos pacientes com triquíase, isso pode ser explicado devido ao fato de as causas que levam os cílios a perderem sua orientação normal e tocarem o olho serem, em geral, decorrentes de patologias crônicas. Outras explicações plausíveis seriam a dificuldade do paciente ter acesso a um profissional especializado e a própria dificuldade de erradicação dos cílios em triquíase.

Mais de uma patologia ciliar pode ser encontrada em uma mesma pálpebra e, provavelmente, o fator etiológico que leva ao aparecimento dessas patologias também é coincidente. Neste estudo, pudemos encontrar associações de triquíase e 


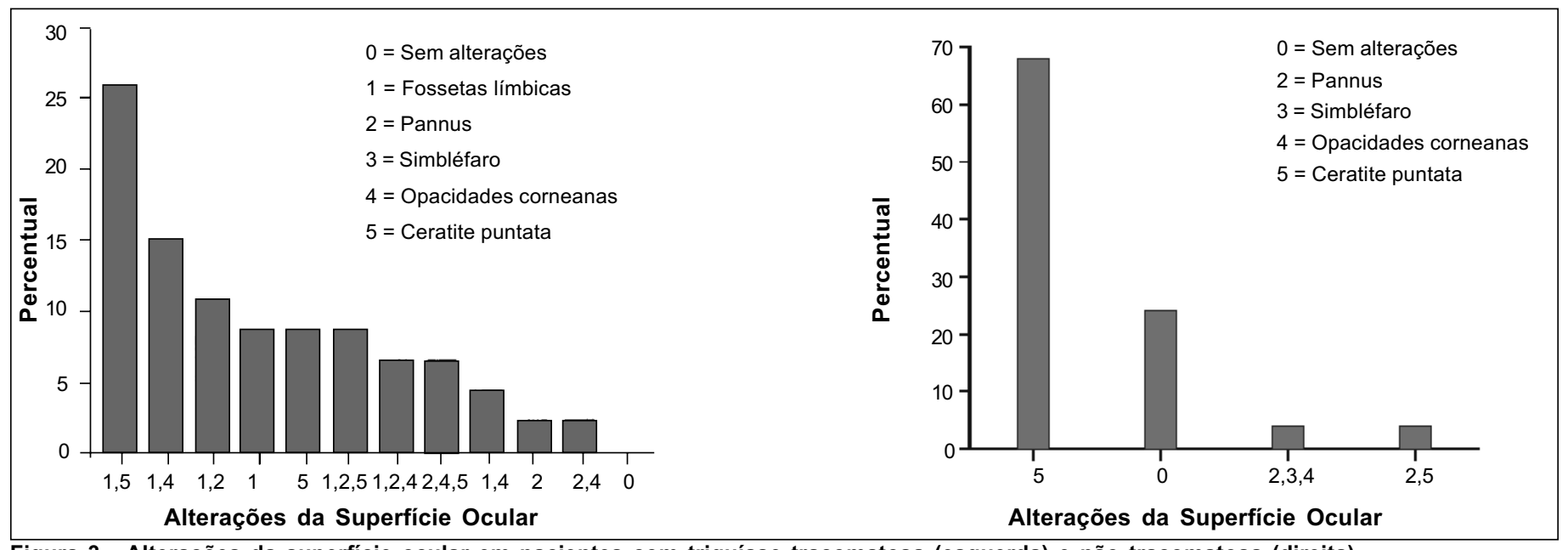

Figura 3 - Alterações da superfície ocular em pacientes com triquíase tracomatosa (esquerda) e não tracomatosa (direita)

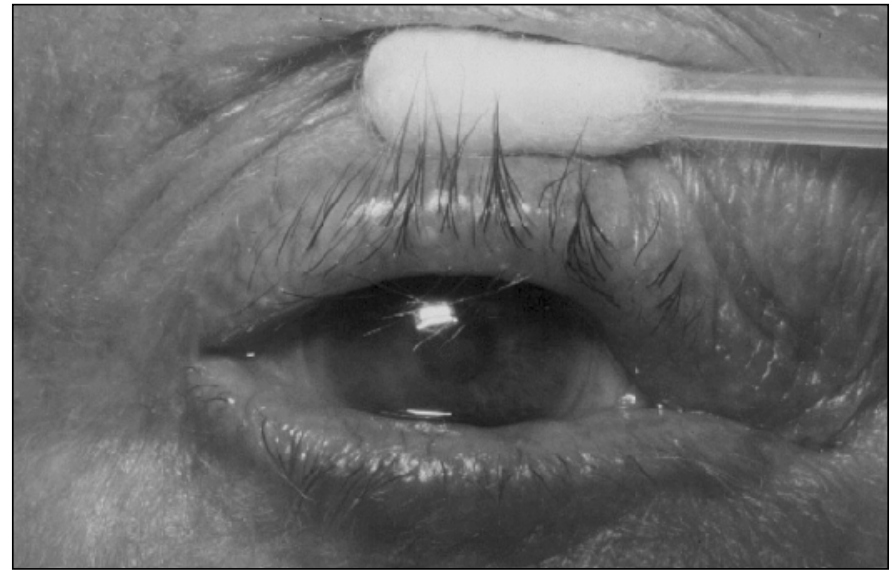

Figura 4 - Cílios mais finos e claros na lamela anterior de pálpebra superior de paciente com triquíase tracomatosa

distiquíase adquirida; triquíase e poliose; triquíase, poliose e ptose ciliar; e madarose e poliose.

O caso do cílio ectópico incarnatum internum (Figura 2), rara patologia congênita, ocorreu em uma criança de 4 anos de idade cuja mãe detectara o cílio patológico no tarso superior de olho direito, quando a criança tinha cerca de um ano e meio. A principal queixa era prurido constante naquele olho e por 2 anos e meio a mãe epilava o cílio pelo menos uma vez a cada 2 ou 3 meses. À biomicroscopia, esse olho apresentava, além do cílio de cor e espessura normais que nascia do tarso a cerca de $2 \mathrm{~mm}$ da margem palpebral, apenas ceratite superior discreta, $\mathrm{o}$ que ratificava o quadro descrito pela mãe. Essa criança fora tratada inicialmente com ressecção em cunha do segmento de tarso onde o cílio emergia, havendo recidiva do mesmo 3 meses após o procedimento cirúrgico. Esse fato nos levou a aventar a hipótese de que apesar de esse cílio emergir da face interna do tarso, seu bulbo ciliar deveria estar presente na pele ou músculo orbicular e, ao invés de crescer direcionando-se para a superfície da pele, provavelmente crescia invertido,

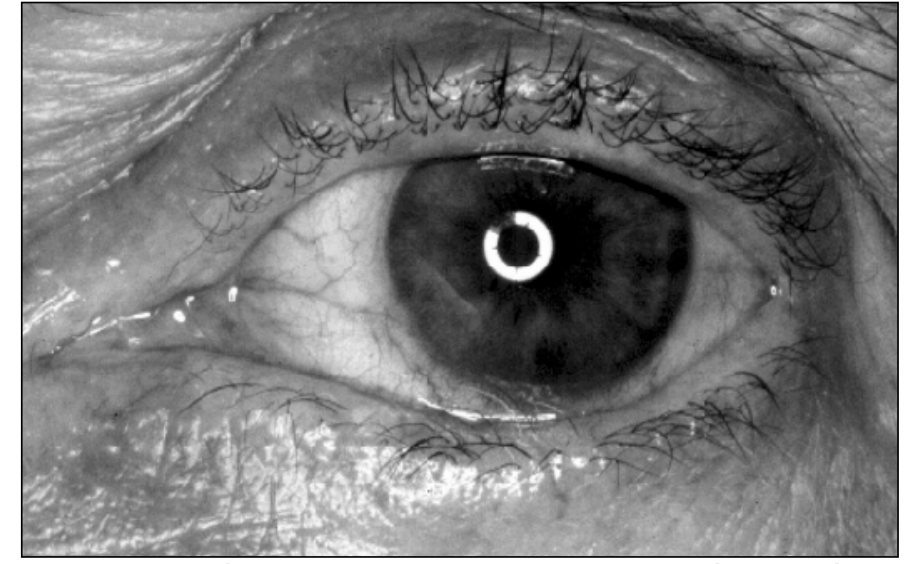

Figura 5 - Triquíase inferior não-tracomatosa. Notar cílio triquiático amelanótico

direcionando-se para o tarso. Sendo assim, um segundo procedimento cirúrgico foi realizado, ressecando-se, em pentágono, todo o segmento palpebral que continha o cílio patológico, não havendo mais recidiva do quadro.

Os outros dois casos de anomalia ciliar não associados à triquíase estiveram associados a enfermidades sistêmicas, sendo que o paciente com poliose isolada tinha doença de Graves e a paciente com madarose ciliar e poliose apresentava Lúpus Discóide com acometimento da margem palpebral, quadro já anteriormente descrito ${ }^{(19)}$. Esse fato indica que, na ausência de mau posicionamento ciliar, anomalias de cor são sugestivas de processo patológico subjacente e, portanto, devem alertar o oftalmologista para um aprofundamento da semiologia. O mesmo pode ser dito em relação à madarose ciliar que sempre esteve associada a outras condições patológicas.

Uma vez que a triquíase foi a patologia ciliar predominante, ela tornou-se objetivo de um detalhamento mais específico.

Várias são as causas que podem levar ao mau direcionamento dos cílios observado na triquíase e já amplamente descritas na literatura. Dentre elas pode-se citar as inflamações 
crônicas da margem palpebral (meibomite, blefarite), dermatoses (elastose actínica, eczema, atopia, hanseníase, herpes zoster), doenças conjuntivais cicatrizantes (tracoma cicatricial, síndrome de Steven-Johnson, penfigóide ocular, queimaduras químicas e físicas) e anomalias cicatriciais da borda palpebral associadas ou não a cirurgias prévias ${ }^{(6,3,17-18,9,20,11)}$.

Neste estudo, a etiologia mais freqüente foi o tracoma, aparecendo em $26(46,4 \%)$ dos 56 pacientes com triquíase. Em outros trabalhos nacionais o tracoma também já foi apontado como a principal causa de triquíase maior ( 5 ou mais cílios acometidos $)^{(9,11)}$ porém não como causa mais importante de triquíase menor (menos de 5 cílios acometidos) ${ }^{(10)}$. Figueiredo e Soares também apontaram o tracoma como a principal causa de triquíase em estudo retrospectivo que avaliou 216 pacientes com essa patologia ${ }^{(9)}$. Provavelmente esse é um achado epidemiológico que deve ser contextualizado regionalmente, uma vez que é nas áreas endêmicas de tracoma que se verifica o maior número de casos de triquíase.

Dois pacientes que apresentaram tracoma como causa da triquíase, também eram portadores de hanseníase. Esses pacientes também apresentavam entrópio de pálpebras superiores e talvez essa associação tracoma-hanseníase explique o achado de casos como esses, de entrópio de pálpebras superiores em pacientes portadores de hanseníase ${ }^{(21)}$.

A magnitude da triquíase foi avaliada não em termos de número de cílios acometidos, mas, sim, em relação ao número de segmentos palpebrais envolvidos (1 segmento, 2 segmentos ou distribuição difusa na pálpebra).

Os cílios em triquíase dispuseram-se de maneira diferente na pálpebra dos pacientes com associação e sem associação com o tracoma, sendo que, nos primeiros, houve maior tendência ao acometimento maior da pálpebra, ou seja, 18 pacientes $(69,23 \%)$ com acometimento de 2 ou 3 segmentos palpebrais e 8 pacientes $(30,77 \%)$ com acometimento de apenas 1 segmento palpebral. Quando o acometimento ocorreu em um único segmento palpebral, houve predominância do segmento médio $(62,5 \%$ dos casos $)$.

Já nos pacientes sem associação com tracoma essa relação foi contrária, predominando acometimento de apenas 1 segmento palpebral, encontrado em 24 pacientes (80\%). Os outros 6 pacientes $(20 \%)$ apresentaram acometimento de 2 segmentos palpebrais, não havendo nenhum caso de acometimento difuso da pálpebra. Esses dados mostram claramente o grande poder de distorção de toda margem palpebral que a conjuntivite tracomatosa possui.

As inflamações crônicas da margem palpebral também foram fator causal de importante parcela dos casos de triquíase (22 casos ou 39,3\%). Figueiredo e Soares e Hirai e colaboradores também apresentaram porcentagens semelhantes em seus estudos sobre triquíase $(34,7 \% \text { e } 36 \% \text {, respectivamente })^{(9,11)}$. Essas taxas são, no entanto, muito variáveis, como indica o trabalho de Creppe \& colaboradores que encontrou apenas $12,6 \%$ de blefarite e/ou meibomite como causa do mau posicionamento ciliar em triquíase menor ${ }^{(10)}$.
Em 18 pacientes (14,3\% dos casos) a causa da triquíase não pôde ser determinada, achado esse ainda um pouco maior que o verificado por Figueiredo e Soares (6\%) e bem diferente do resultado do estudo sobre triquíase menor de Creppe e colaboradores $(66,1 \%)^{(9-10)}$. Como o mesmo trabalho ${ }^{(10)}$ identificou um número bem menor de inflamações crônicas da margem é possível que essas disparidades digam respeito aos critérios utilizados para o diagnóstico de inflamação marginal.

Em relação à lateralidade, em 40 pacientes $(71,4 \%)$ a triquíase foi bilateral e em $16(28,6 \%)$ foi unilateral, resultado que mais uma vez se assemelhou ao de Figueiredo e Soares (70,83\% bilateral e $29,17 \%$ unilateral), mas que contrastou com os achados de Dantas que mostrou bilateralidade em $18,8 \%$ e unilateralidade em $81,2 \%$ em um estudo de 32 pacientes portadores de triquíase ${ }^{(9,18)}$. Esse achado é explicado pelo fato de o tracoma ter sido o principal fator etiológico da triquíase, uma vez que a doença age de forma semelhante em ambos os olhos, proporcionando maior bilateralidade no aparecimento da triquíase.

Não houve diferença significativa de acometimento pela triquíase em relação ao olho direito (46 pacientes) e ao olho esquerdo (49 pacientes), assim como mostraram Creppe e colaboradores $^{(10)}$.

Quando analisamos, entretanto, pálpebra superior e pálpebra inferior separadamente, houve diferenças significativas quando a triquíase esteve ou não associada ao tracoma. Sendo assim, dos 26 pacientes com triquíase e tracoma, o acometimento palpebral foi predominantemente superior (22 pacientes $84,6 \%$ dos casos), enquanto que nos 30 pacientes com triquíase não associada ao tracoma o acometimento palpebral foi predominantemente inferior (28 pacientes - 93,3\% dos casos). Esses resultados mostram a influência da tarsite tracomatosa na localização da triquíase associada ao tracoma, uma vez que esse processo aparece predominantemente na pálpebra superior. Já nos pacientes com triquíase não associada ao tracoma, os fatores agressores (sol, poeira, vento, poluição) talvez atuem predominantemente nas pálpebras inferiores.

Um importante resultado deste trabalho, e que se assemelha a outros da literatura, é o fato de a triquíase estar associada a dano corneano em grande número dos olhos acometidos (84 dos 96 olhos com triquíase, ou seja, $87,5 \%$ dos casos $)^{(22,20,7)}$.

Como era de se esperar, o dano à superfície ocular foi mais grave e com predominância de deformidades profundas e definitivas (fossetas límbicas, pannus e opacidades corneanas) nos olhos dos pacientes com triquíase associada ao tracoma (Figura 3), já que, nessa enfermidade, o mesmo processo inflamatório crônico que leva ao aparecimento da triquíase leva também a profundas transformações nos demais componentes do olho como filme lacrimal, conjuntiva e tarso.

A ceratite puntata foi a alteração corneana mais freqüentemente encontrada ( 66 dos 96 olhos com triquíase - 68,75\%), o que também ocorreu em outro trabalho em que foi estudada a triquíase menor ${ }^{(10)}$. Esse achado condiz com a principal queixa apresentada pelos pacientes, uma vez que as microerosões corneanas, provocadas pelo toque dos cílios patológicos, 
leva à exposição dos filetes nervosos da córnea, produzindo a sensação de corpo estranho.

Enfim, é importante ressaltar que algumas características ciliares morfológicas utilizadas para a definição de distiquíase, como cílios finos e mais claros ${ }^{(14,3)}$. foram também verificadas nos casos de triquíase associada ou não ao tracoma (Figuras 4 e 5). Esses dados mostraram de maneira clara que a distinção entre triquíase e distiquíase não pode ser feita por características morfológicas dos cílios. Assim, pode-se sugerir que outros critérios, como por exemplo a localização do cílio acometido na margem palpebral, possam ser utilizados para diferenciar triquíase de distiquíase: triquíase, cílios na lamela anterior, ou seja, anteriormente à linha das glândulas de Meibomius; distiquíase: cílios na lamela posterior, ou seja, no nível ou posterior à linha das glândulas de Meibomius.

\section{ABSTRACT}

Purpose: To describe and analyze the eyelash abnormalities diagnosed at the Hospital of the School of Medicine of Ribeirão Preto. Methods: A total of 59 patients who presented during 1999 with eyelash problems at the oculoplastic clinic were studied. Patients with previous treatments were excluded. Data collection included systemic or local disease, symptom duration, eyelid and eye surface abnormalities as well lash morphologic and location characteristics. Results: Trichiasis was the main diagnosed abnormality ( $94.9 \%$ of the cases). Trachoma was the main disease associated with trichiasis. Trachomatous trichiasis differed from nontrachomatous trichiasis according to the number of affected eyelid segments, laterality, affected eyelid (upper or lower) and magnitude of corneal damage. Lash abnormalities regarding color, size and thickness were seen in both trachomatous and nontrachomatous trichiasis. Conclusions: Trachomatous trichiasis continues to be the main lash problem in the Hospital of the School of Medicine of Ribeirão Preto. Color and thickness lash abnormalities can not be used as criteria for the distinction between trichiasis and acquired distichiasis.

Keywords: Eyelash; Trachoma; Eyelid diseases; Eyelid

\section{REFERÊNCIAS}

1. Netland PA, Sugrue SP, Albert DM, Shore JW. Histopathologic features of the floppy eyelid syndrome. Involvement of tarsal elastin. Ophthalmology 1994;101:174-81.

2. Guimarães FC, Cruz AAV. Eyelid changes in long-standing leprosy. Ophthal Plast Reconstr Surg 1998;14:239-43.

3. Duke-Elder S, MacFaul PA. Disorders of the eyebrows and lashes. Em: DukeElder S. System of Ophthalmology. London: Henry Kimpton; 1974. p.373-90.

4. Fledelius HC, Stubgaard M. Changes in eye position during growth and adult life as based on exophthalmometry, interpupillary distance and orbital distance measurements. Acta Ophthalmol 1986;64:481-6.

5. Elder MJ. Anatomy and physiology of eyelash follicles: relevance to lash ablation procedures. Ophthal Plast Reconstr Surg 1997;13:21-5.

6. Scheie H, Albert DM. Distichiasis and trichiasis: origin and management. Am J Ophthalmol 1966;61:718-20.

7. Yung CW, Massicotte SJ, Kuwabara T. Argon laser treatment of trichiasis: a clinical and histopatological evaluation. Ophthal Plast Reconstr Surg 1994;10:130-6.

8. Figueiredo ARP, Soares EJC, Dantas RRA. Triquíase. In: Soares EJC, Moura EM, Gonçalves JOR. Cirurgia e plástica ocular. São Paulo: Rocca; 1997. p.185-92.

9. Figueiredo ARP, Soares EJC. Trichiasis: diagnosis and management. Orbit 1992;11:137-46.

10. Creppe MC, Silva MRBM, Schellini SA. Tratamento por eletrólise do mal posicionamento dos cílios palpebrais. Rev Bras Oftalmol 1996;55:35-9.

11. Hirai FE, Shiguematsu AI, Schellini SA, Padovani CR. Tratamento cirúrgico da triquíase maior. Rev Bras Oftalmol 1998;57:357-61.

12. Cruz AA, Menezes FA, Chaves R, Pinto Coelho R, Velasco EF, Kikuta H. Eyelid abormalities in lamellar ichtyoses. Ophthalmology 2000;107:1895-8.

13. Dalgleish R. Ectopic cilia. Brit J Ophthalmol 1966;50:592-4.

14. Duke-Elder S. Developmental anomalies of the eyelids. Em: Duke-Elder S. Text-book of ophthalmology. London: Henry Kimpton; 1952. p.4631-93.

15. Satterfield D. Pediculosis. In: Mannis MJ, Macsai MS, Huntley AC. Eye and skin disease. Philadelphia: Lippincott-Raven; 1996. p.607-10.

16. Shields JA, Shields CL. Hair follicle tumors. In: Shields JA, Shields CL. Eyelid and conjunctival tumors. Philadelphia: Lippincott Williams \& Wilkins; 1999. p.64-73.

17. Barber K, Dabbs R. Morphological observations on patients with presumed trichiasis. Brit J Ophthalmol 1988;72:17-22.

18. Dantas RRA. Triquíase-fotocoagulação com laser de Argônio. Rev Bras Oftalmol 1992;51:9-12.

19. Huey C, Jakobiec FA, Iwamoto T, et al. Discoid lupus erythematosus of the eyelids. Ophthalmology 1983;90:1389-98.

20. Oshry T, Rosenthal G, Lifshitz T, Shani L, Yassur Y. Argon freen laser photoepilation in the treatment of trachomatous trichiasis. Ophthal Plast Reconstr Surg 1994;10:253-5.

21. Moreno RD, Woods WJ. Prevalência das alterações oculares em pacientes portadores de hanseníase em um hospital colônia no Acre. Arq Bras Oftalmol 1999;62:254-7.

22. Sbrissa RA, Sbrissa CA, Fernandes JM. Correção cirúrgica da triquíase. Rev Bras Oftalmol 1991;50:57-9.

\title{
ABO ELETRÔNICO
}

\author{
Novo site
}

Acesso: http://www.abonet.com.br 